\title{
Under-recording of diabetes on medical record may be associated with adverse in-hospital outcomes
}

\author{
Rogerio Silicani Ribeiro*, Marcia Nery, Jose Antonio Maluf de Carvalho, Ana Claudia Latronico, \\ Magda Tiemi Yamamoto, Thalita Barrera Modena, Adriana de Fatima Avansi
}

From 20th Brazilian Diabetes Society Congress

Porto Alegre, Brazil. 11-18 November 2015

\section{Background}

Hospitalized patients with diabetes (DM) and stress hyperglycemia $(\mathrm{SH})$ have higher risk of complications and mortality compared to normal (NL), especially if hypo or hyperglycemia come about. The under-recording of diabetes may difficult the recognition of glucose abnormalities, delay adequate therapy and aggravate prognosis of non-registered diabetics (DMNR).

\section{Objective}

To compare in-hospital outcomes among patients with DMNR, DM, SH and NL.

\section{Materials and methods}

This is a retrospective analysis including 62136 adults (> 18 yrs.) admissions in groups NL (69\%), DM (17\%), DMNR (5\%), and SH (9\%), hospitalized between 2010 and 2013. Patients were classified according to five coded discharge diagnoses registered in electronic medical record. The inclusion criteria for DMNR were registration of DM in previous admissions and omission of diagnosis in consecutive readmission. Inclusion criteria were length of stay between 2 and 120 days, availability of capillary glucose (at least one measurement for NL patients and two measurements for DM, DMNR and $\mathrm{SH}$ patients). Exclusion criteria were pregnancy. In hospital outcomes included in the retrospective analysis were nosocomial infection, sepsis, intensive care unit admission and death. To compare the proportion of adverse outcomes we perform chi-square test.

\section{Results}

Average age was 54,8 yrs., the proportion of females was $52 \%$. The proportion of hospitalizations with surgical interventions was $44 \%$. The average length of stay among NL, DM, DMNR and SH, were 4,7; 7,3; 13,9 and 17,1 days respectively. The incidence of nosocomial infection were $0,4 \% ; 0,9 \% ; 2,3 \%$ and $3,9 \%$ and the incidence of sepsis were $1,5 \% ; 4.0 \% ; 13,7 \%$ and $16 \%$ in NL, DM, DMNR and SH, respectively. The mortality rate were $0,8 \%, 1,4 \%, 10,2 \%$ and $12,7 \%$, respectively. P values were $<0.05$ for every outcome described.

\section{Conclusion}

The omission of the diagnosis of DM on medical record is associated with adverse in-hospital outcomes.

Published: 11 November 2015

doi:10.1186/1758-5996-7-S1-A96

Cite this article as: Ribeiro et al:: Under-recording of diabetes on

medical record may be associated with adverse in-hospital outcomes.

Diabetology \& Metabolic Syndrome 2015 7(Suppl 1):A96.

\footnotetext{
* Correspondence: dr.rogerioribeiro@uol.com.br

Universidade Federal de São Paulo, São Paulo, Brazil
} 